\title{
AN EXPERIMENTAL INVESTIGATION ON THE COALESCENT BEHAVIORS OF COLLIDING DROPLETS
}

\author{
C.H. Wang * K.L. Pan * S.Y. Fu ${ }^{* *} \quad$ W.C. Huang ** \\ Department of Mechanical Engineering \\ National Taiwan University \\ Taipei, Taiwan 10617, R.O.C.
}

\author{
J.Y. Yang * \\ Institute of Applied Mechanics \\ National Taiwan University \\ Taipei, Taiwan 10617, R.O.C.
}

\begin{abstract}
The coalescent behaviors in collisions between two droplets respectively made of different alkanes, water and alkane, methanol and alkane, and ethanol and hexadecane were experimentally studied. The coalescent results between two droplets of different alkanes are qualitatively the same as that with the same material, which simply form a spherical droplet. However, it took time to have the concentration within the droplet to become uniformly distributed. The collision results of water and alkane droplets collision become slightly more complex, in most cases, the water droplet was either inserted into or adhesive to the hexadecane droplet while only insertion was observed if the target droplet was dodecane or heptane. The inserted water droplet tends to partially expose to the environment as the volume fraction of water is sufficiently high, say, $\sim 0.62$ for hexadecane, $>0.70$ for dodecane, and $>0.78$ for heptane; and the limit is lowered with the decreasing of water or merged droplet size. For the cases of methanol and alkanes, and ethanol and hexadecane, the two colliding droplets were adhesive to each other in all the studies. Furthermore, in most conditions, air bubbles were observed immediately after the collisions, while only few or even none of them might be trapped within the final merged droplet.
\end{abstract}

Keywords : Droplet collision, Insertive coalescence, Adhesive coalescence.

\section{INTRODUCTION}

Motivation for the present investigation came from the confluence of two phenomena relevant to droplet and spray combustion, namely, the combustion and microexplosion of single droplet of multi-component fuels and the coalescence and bouncing of two colliding droplets.

Regarding the background for miscible multicomponent droplet combustion, we note that the studies $[1,2]$ are of significant technical interest because most practical liquid fuels are blends of many chemicals each characterized by its own physical-chemical properties such as volatility, diffusivity, and reactivity. In particular, a phenomenon termed as microexplosion that violently ruptures the burning droplets has been largely observed in the experiments [3 7].

Ever since Hopkinson [8] sprayed water into the combustion chamber and successfully minimized knock, there have been substantial interests and hence researches into the possibility of using water to modify the performance of diesel engines. Among those, the use of emulsions has been shown [9 11] to give the most effective way to improve the combustion characteristics. Fundamental studies [12 15] then suggested that microexplosion was responsible for the improvement.
The inhomogeneous nature of miscible multicomponent and immiscible water/diesel emulsions may aid the rupture of droplets during the burning. To comprehend the intensified mechanism, it is interesting to know what would be the collision consequence of directly mixed droplets formed through the collision between liquid jets or droplets. However, previous studies such as those in [16-18] are concerned with the same single-component liquid and there is a lack of information for the collision between different liquids.

Our latest investigations [19 21] reported the burning behaviors of collision-mixed droplets, which showed the potential of getting violent rupture of the burning droplets. In this report, tasks are then more focused on the detailed droplets collision behaviors during the merging process and the final coalescent outcome, with different liquid characteristics.

In the next section the experimental specifications on the study will be first introduced, which will be followed by the results for the collision-mixed droplets.

\section{EXPERIMENTAL APPARATUS AND TEST PROCEDURES}

The modified ink-jet printing technique for droplet generation, by which the droplet is generated on de-

* Professor ** Graduate student 
mand, used in Wang and Chen [15], was adopted in the study. In the approach, a rectangular pulse of proper amplitude and width was applied on a circular piezoelectric crystal plate, and the deformation of plate squeezed out a tiny amount of incompressible liquid supplied by a reservoir through a fine glass nozzle. The ejected liquid then formed a droplet through its surface tension.

Two such generators were used, each with its own pulse amplitude, width, and phase but with identical pulse rate. Thus, two stable droplet streams, with equal or different sizes, at the same generation rate, can be simultaneously and independently obtained.

The crucial requirement is to have the two streams of fine droplets collide properly. The two droplet generators were independently hanged on the rotational arms and then separately seated on $x-y$ translation stages. By rotating the arms and adjusting the $x-y$ stages, the trajectories of the two streams of droplets could fall into a point on the same vertical plane. Afterward, varying the phase between the two generators, the two streams of droplets could then collide in a desired manner - head-on or off-centered collisions.

A strobe was synchronized with that of generated droplets while variable phase shift could be adjusted, so that the images of the droplets moving along their trajectories before and after collision were actually frozen. The resolution of the shifting time was set to be 0.1 millisecond in the present study, which is equivalent to the use of a high-speed movie camera at $10000 \mathrm{fps}$ and can be further enhanced by changing the resistances and/or capacitances of the phase shifting circuit.

Enlarged images were obtained by a CCD camera attached with a Baush \& Lomb mono-zoom-7 long focus microscopic lens. The uncertainty of determining the size of the droplets was about 2, mainly from reading the boundary of the droplet image.

The droplet size was mainly controlled by the exit size of the glass nozzle, while the pulse amplitude and width of the generator had a minor effect. In the study, the size of the droplet was varied from about 170 to $380 \mu \mathrm{m}$, which gave quite a wide range of mixing ratios.

By measuring the variation of the droplet positions on two consecutive photos with known shifting time, the droplet velocities could then be calculated accordingly, and the relative colliding velocities were ranged from 0.3 to $4.0 \mathrm{~m} / \mathrm{s}$ in most of the study.

Based on the mixing potential between different types of liquids, the presentation is divided into collisions of miscible droplets and that of immiscible droplets. For miscible liquids, the collisions were made between two droplets of different alkanes. For immiscible liquids, it was further classified as collisions of droplets respectively made of water and alkane, methanol and alkane - where hexadecane, dodecane, decane and heptane among the alkane family were chosen for the test, and ethanol and hexadecane.

\section{RESULTS AND DISCUSSION}

In most of the previous studies on droplet collision [16 18], only the same single-component liquid for both droplets was used; the droplets are thus miscible. As such, it was found that the results could be classified into several distinct regimes with respect to three important parameters, the collision Weber number $W e, W e$ $=\rho\left(d_{1}+d_{2}\right) U^{2} / 2 \sigma$, the impact number $B, B=2 \chi /\left(d_{1}+\right.$ $d_{2}$ ), and the ratio of droplet diameter $\Delta, \Delta=d_{2} / d_{1}$, where $d_{1}$ and $d_{2}$ are the diameters of the colliding droplets, $U$ the relative velocity at collision, $\rho$ and $\sigma$ the density and surface tension of the liquid respectively, and $\chi$ the projection of the separation distance between the droplet centers in the direction normal to that of $U$, or so-called off-center distance thereafter. For water and alkane droplets, the typical schematic diagrams of the various collision regimes can be found in [16]-[18], which show that the collision outcomes of the binary droplets can be characterized as permanent coalescence, bouncing, and temporary coalescence followed by separation either associated with or without concomitant satellite droplets.

For all the studies in the following sections, with $\chi \approx$ 0 (head-on collision), the permanent coalescence was the only collision outcome under the test conditions; and the temporary coalescence followed by separation occurred as increasing of the off-center distance at a given collision velocity. Nevertheless, it should be pointed out that the present study does not intend to quantitatively designate the boundary of the regimes but only qualitatively clarify the phenomena of the permanently coalescent droplets. Moreover, for the collision between different liquid components, the individual $\rho$ and $\sigma$ of the species let the Weber number not uniquely be defined.

\subsection{Miscible Fuels: Collisions Between Alkane Droplets}

Collisions of droplets with different alkanes, individually generated by two streams of droplets, are investigated in this section. They are further compared with the experiments for the colliding droplets with the same alkane. Figure 1 shows the photographic images of typical coalescent collision sequences between two droplets with the same component and Fig. 2 shows the images between heptane and hexadecane droplets; and each includes both (a) head-on and (b) off-center collisions, respectively.

Typically, for a normal, spherical droplet, the strobe light transmits directly through the center region but deflects around the edge, such that the droplet image appears to be brighter in the center than that at the edge regions, which can be easily seen from the recorded photos before collision in both Figs. 1 and 2 . The contrast of the photo image is affected by the orientation of the light source; thus the interior appearance of a spherical droplet image may not be well symmetric. 


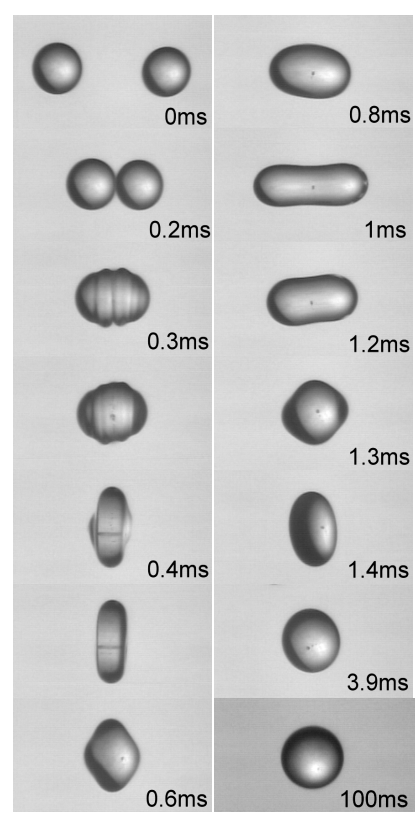

Left - $256 \mu \mathrm{m}$
Right - 255
head - on

(a)

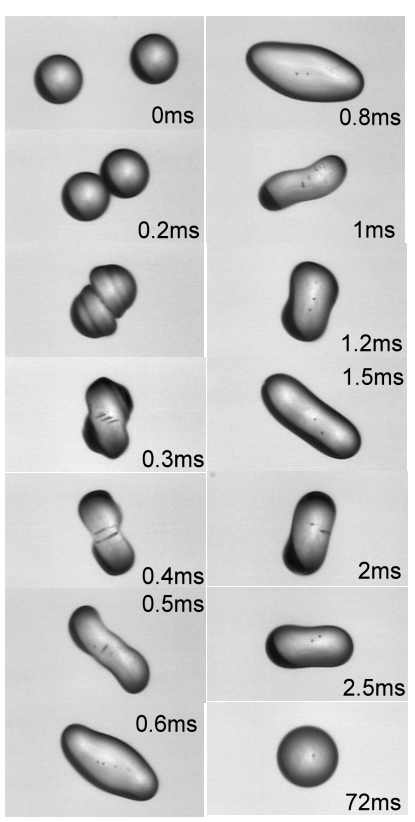

Left $-256 \mu \mathrm{m}$

Right - 255

off - center

(b)

Fig. 1 Sequential photos of the formation of collisioncoalescent droplet, the same component

Immediately after the collision, the shape of the coalescent droplet is irregular, so is the recorded image of its light contrast. However, the effect is noticeable only when the curvature of the boundary of the coalescent droplet varies substantially. For the colliding droplets with the same liquid, the duration of the irregularity of light contrast sustained only within a millisecond and back to normal thereafter, even though the coalescent droplet was not in final spherical shape yet. One can read it from the sequential photos in Figs. 1(a) and 1(b), which respectively represent the results of head-on and off-center collisions, with the same liquid.

For colliding droplets constituted of different liquids, the variation of refractive index due to the non-uniform distribution of species concentration then gives an additional factor to have the recorded images shadowed. This would last for tens of millisecond, and the droplet had already stabilized in sphericity. For head-on collisions, the increase of the collision velocity increased the mixing, thus shortened the period with appearance of shadowness. For off-center collisions, increasing of the impact number decreased the mixing and thus lengthened the duration. By reading the variation of image contrast, one can approximately know the mixing processes. Figures 2(a) and 2(b) respectively give the results of head-on and off-center collisions with different components. One can easily differentiate the difference between Figs. 2 and 1. Figures 3(a) and 3(b) respectively show the interior mixing patterns for headon and off-center collision, and a regular rotated flow pattern was observed for off-center collision.

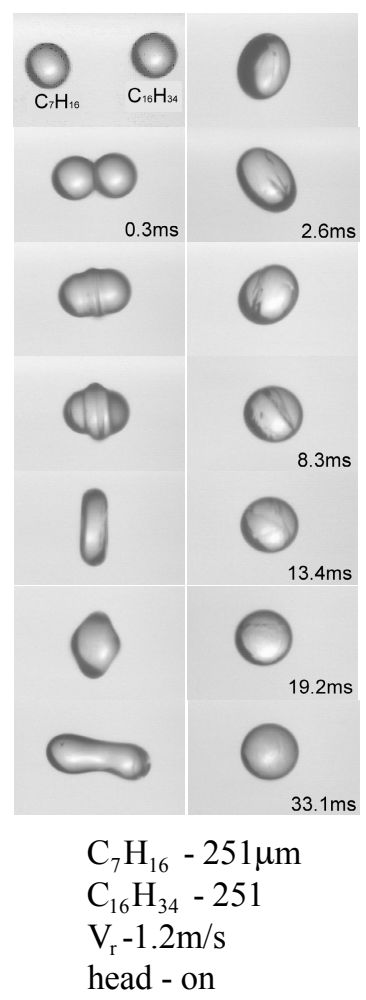

(a)

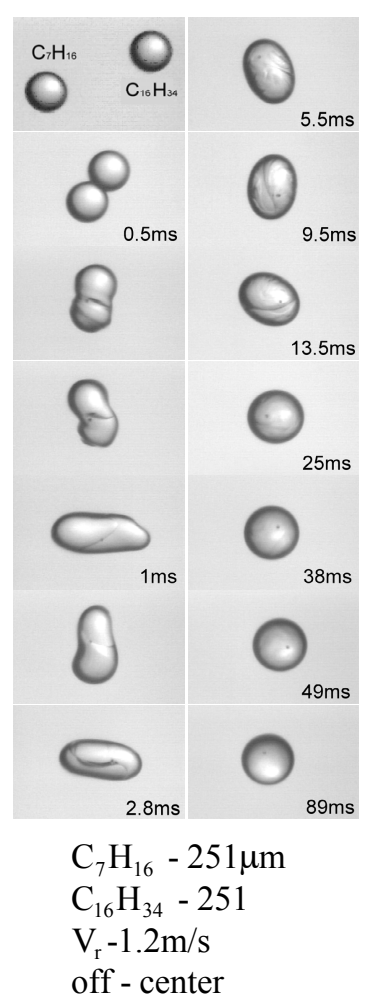

(b)
Fig. 2 Sequential photos of the formation of collision-coalescent droplet, different components

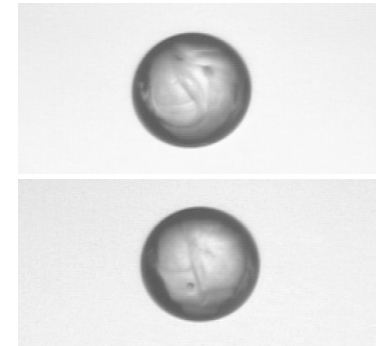

(a) head-on

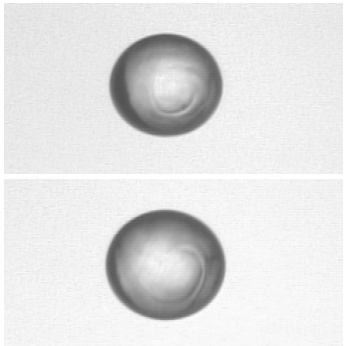

(b) off-center
Fig. 3 Sequential photos of the formation of collision-coalescent droplet, different components

Carefully examining the collision images of Figs. 1 and 2, or the enlarged photos of Fig. 4, a phenomenon that is of particular interest in addition to the spatial non-uniformity of light contrast is the presence of one or more dark spots within the merged mass. It occurred for collisions involving equal or unequal size, head-on or off-center orientation, and the same or different components of droplets. The presence of these dark spots were also observed in other experiments [19 21], which was suggested in [19] as air bubbles trapped within the merged mass.

Almost in all cases of the study, fine spots appeared immediately after the collision. The spots could be small liquid droplets together with tiny air bubbles. As the mixing process went on, the fine liquid droplets dissolved into and mixed with the nearby liquid, thus disappeared within millisecond. Compared with the 


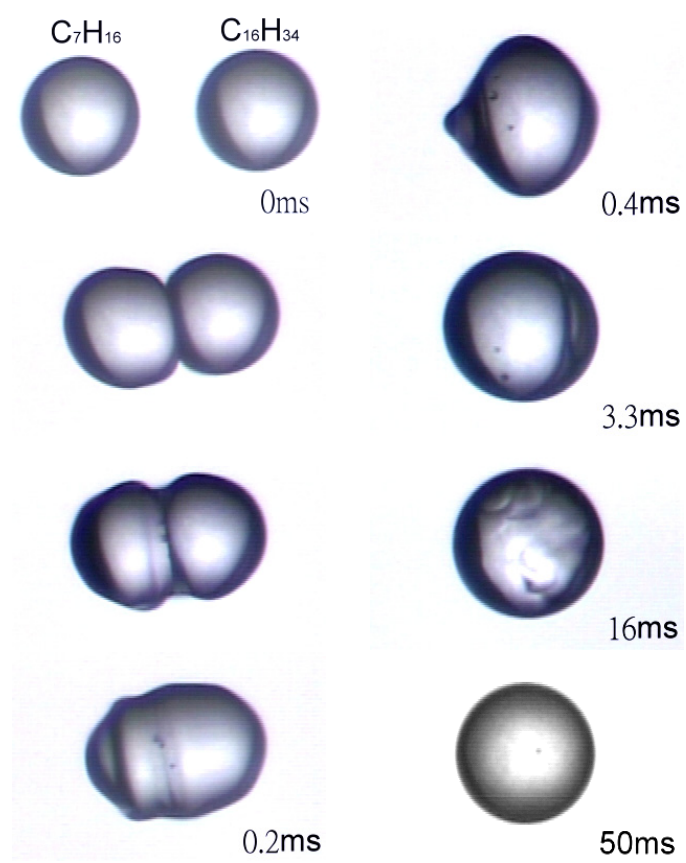

Fig. 4 Enlarged photos to show the formation of tiny spots [20]

liquid-liquid dissolution, air bubbles are much more difficult to dissolve and have higher preference to sustain. The disappearance of the tiny air bubbles was possible due to either the coalescence into bigger bubbles or convection to the droplet exterior, led to only few or even none dark spots within the coalescent mass after the completion of the merging process.

In the experiment, statistically, the observed results showed that the probability of having air bubbles trapping in the final merged mass depended on the relative collision velocity and orientation, and the selected liquids of droplets. The preliminary results showed that at a fairly low relative collision velocity, air bubbles might be completely eliminated afterward. Increasing the relative velocity and/or the off-center distance tended to increase the probability of trapping air bubbles, so is for the collision droplets with the same component. There was no clear evidence that the sizes of collision droplets would affect the final exhibition of air bubbles. More sophisticated and systematic experiments should be conducted to clarify those observations.

It should be emphasized that, due to high repeatability and stability of the droplet streams in the experiment, the recorded collision images presented actually represented thousands of coalescent droplets under the same collision conditions.

\subsection{Immiscible Liquids: Collisions Between Water and Alkane Droplets}

Since it is known that alkane fuel and water are not miscible, it was anticipated that coalescence of the droplets was not possible and bouncing should be the only outcome after collision. It was thus surprising that

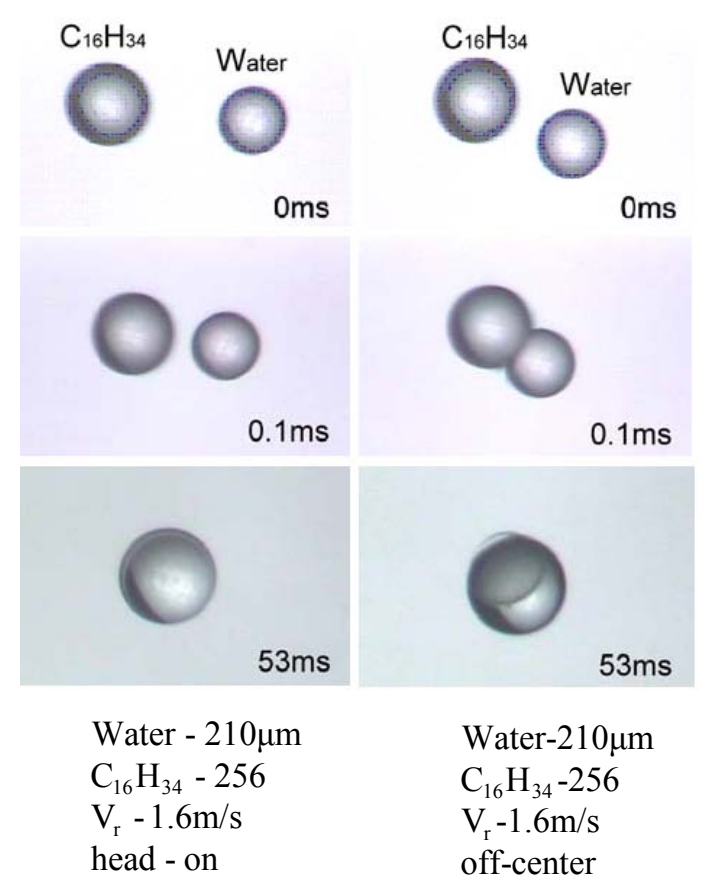

(a) insertive

(b) adhesive

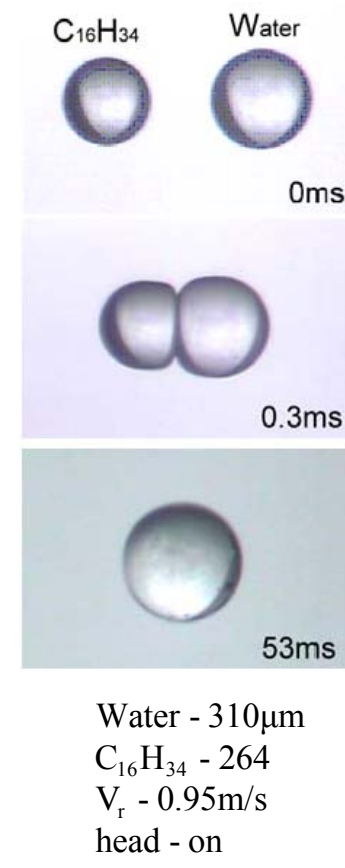

(c) insertive

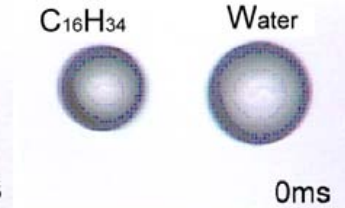

Oms

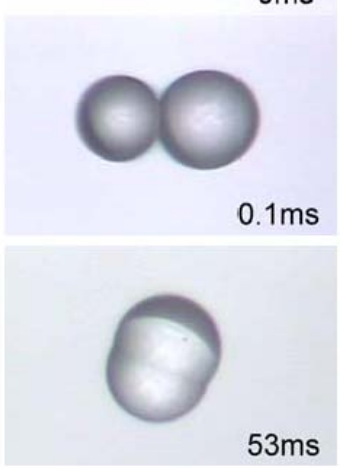

Water $-310 \mu \mathrm{m}$

$\mathrm{C}_{16} \mathrm{H}_{34}-264$

$\mathrm{V}_{\mathrm{r}}-2.1 \mathrm{~m} / \mathrm{s}$

head - on

(d) adhesive
Fig. 5 Collision-coalescent droplet, water and hexadecane

coalescence of the colliding droplets made of water and hexadecane respectively was actually observed [20]. The results showed that the coalescence could be further divided into two modes, namely, the insertive coalescence and adhesive coalescence. For the insertive coalescence, since the surface tension of water is much larger than that of hexadecane, the water droplet was swallowed into the interior of the hexadecane droplet, or the hexadecane liquid was spread on top of the integrated water droplet, and hence the water droplet was 
enclosed by hexadecane. As for adhesive coalescence, the colliding droplets appeared to be in contact with each other and a boundary indicating a contact surface clearly existed. Figures 5(a) and 5(b) respectively show the typical images of the insertive and the adhesive coalescent droplets. It was also suggested [20] that the temperature appears to be a dominating factor to yield the specific mode of coalescence of water/hexadecane droplet through the variation of surface tension, and the adhesive coalescent water/hexadecane droplets changed to insertive coalescence at elevated environmental temperature under most of their test conditions.

In this study, it was observed that the two modes existed for most of the test droplet sizes, exclusive of very high water volume fraction. In general, as the relative collision velocity is high enough, the coalescent droplet was in insertive mode as the impact momentum of water droplet can overcome the surface tension of hexadecane. By increasing the off-center distance, however, the adhesive mode was preferred by the droplet coalescence. The difference can be identified in Figs. 5(a) and 5(b).

It is not surprising that, by further increasing the collision velocity of a head-on collision, the water droplet can penetrate hexadecane fuels, and the insertively coalescent droplet might change to adhesive merging mode. Figures 5(c) and 5(d) then respectively show the typical images of the mode changing, by increasing the collision velocity. Experimental results also show that a higher velocity is needed for the mode changing in the collision associated with a smaller water droplet, because the required high velocity is beyond our test limits, thus we do not have the mode changing for the smaller water droplet size as that in Fig. 5(a).

It was also observed in [20] that there is no droplet ignition as the water volume fraction of the coalescent water/hexadecane droplets is higher than $\sim 0.54$, and was suggested that the behavior is attributed to the higher heating needed for the increasing of water droplet size. However, some new evidence in the present study shows that as the volume fraction of water within the coalescent droplet was sufficiently high, the adhesive merging might be the only existing mode, even at the elevated temperature. The consequence, evaporation of the exposed water droplet, made the ignition delay much prolonged, or even with no ignition.

Figure 6(a) shows the head-on collision of water and hexadecane droplets with sizes of 279 and $236 \mu \mathrm{m}$ respectively, for which the corresponding water volume fraction was $\sim 0.62$. It can be seen from the figure that the water droplet was not enclosed by hexadecane fuel and the merged droplet was in adhesive mode. Actually, it was always in adhesive mode for all tested collision velocities and orientations. On the other hand, the case in Fig. 6(b) (a re-plot of Fig. 5(c)), which was associated with almost the same water volume fraction but with larger sizes of water and hexadecane droplets, i.e. 310 and $264 \mu \mathrm{m}$, was in the insertive merging mode. It could change to an adhesive mode when the velocity is increased, as shown in Fig. 5(d).

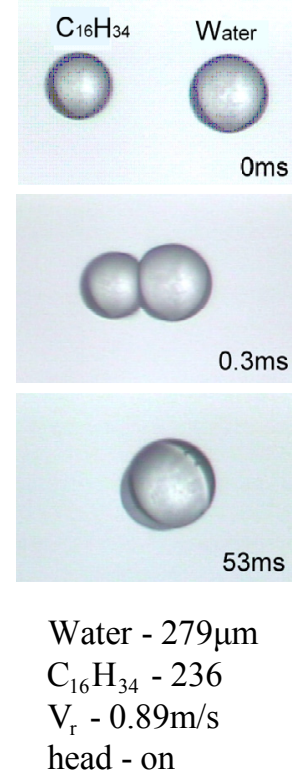

(a) adhesive

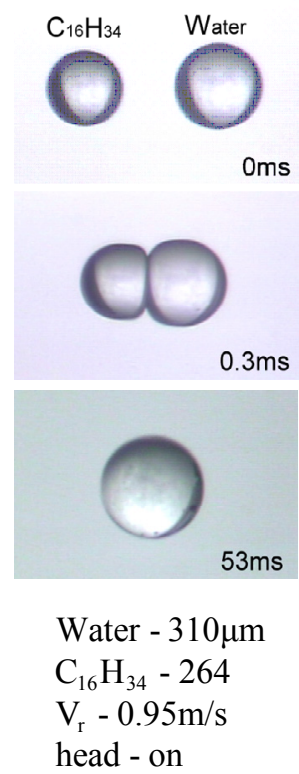

(b) insertive
Fig. 6 Collision-coalescent droplet, water and hexadecane

Comparing the two cases, with the same water volume fraction $\sim 0.62$, the estimated thickness of hexadecane layer on the uniformly enclosed water droplet are $\sim 23.8$ and $27 \mu \mathrm{m}$, respectively for water size of 279 and $310 \mu \mathrm{m}$, and the difference is remarkable. The surface tension force then plays an important role to break the thin surface layer of $23.8 \mu \mathrm{m}$, such that the interior water droplet will always partially expose to the environment. As a consequence, the exposure of inner water droplet might lead the coalescent droplets with high water volume fractions in [20] away from the ignition. Furthermore, the present results suggested that the size of the water or the final merged droplet is also a major factor to affect the lower limit of water volume fraction characterized (or classified) by permanent water exposure. More investigations are needed to systematically define the limits.

For the collisions of water/heptane droplets, only insertive coalescence was observed under all the test conditions (up to $\sim 0.78$ of water volume fraction, with estimated surface layer thickness $\sim 10 \mu \mathrm{m}$ ). The water droplet was completely enclosed by heptane, for both head-on and off-center collisions at different impact velocities. Figures 7(a) and 7(b) respectively show the sequential images of head-on and off-center collisions for water/heptane droplets. The results were quite similar to that of water/dodecane collisions, up to $\sim 0.7$ of water volume fraction and with estimated surface layer thickness $\sim 15 \mu \mathrm{m}$, in which only insertive coalescence was observed.

From these arguments, it might be deduced that the surface tension of alkanes plays an important role in those different coalescent behaviors for a given collision condition. And, present findings should be valuable to the practical usage of fuels such as diesel and kerosene, since their major components are made of alkanes. 


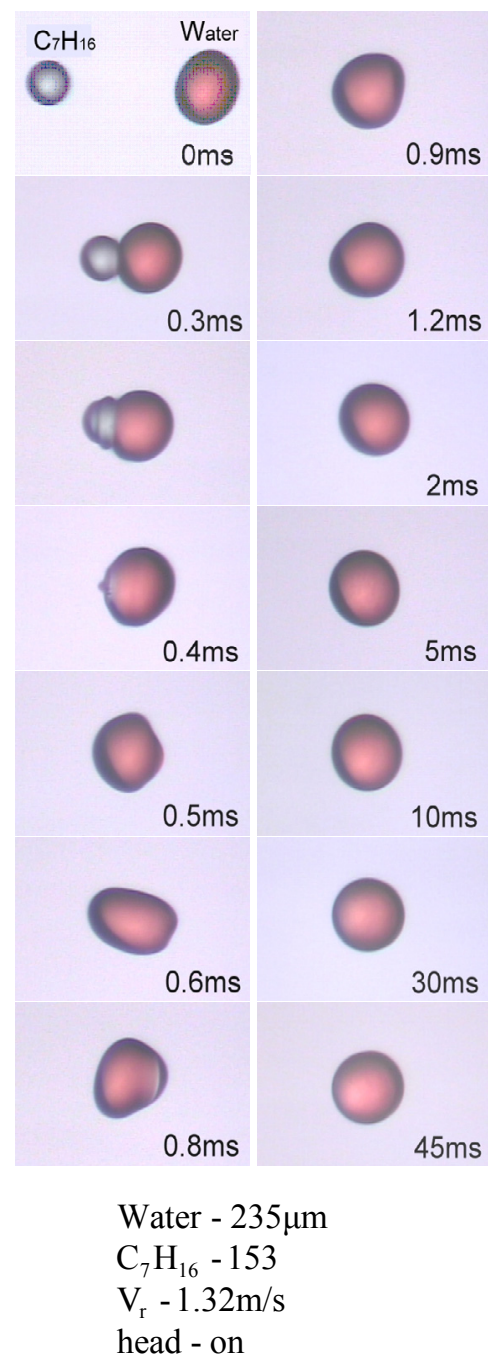

(a)

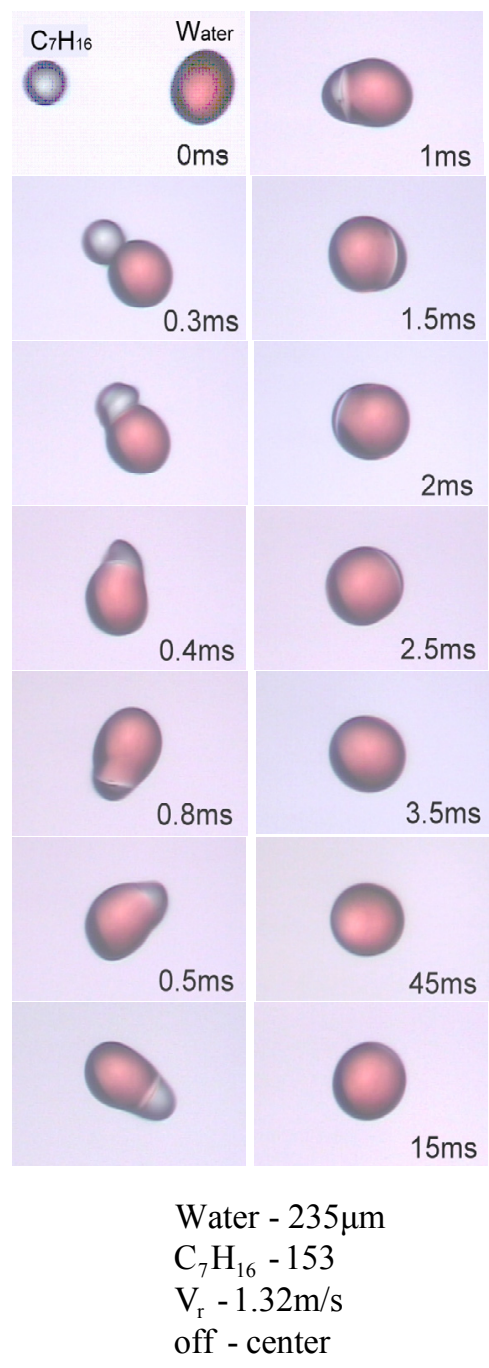

(b)

Fig. 7 Sequential photos of the formation of collision-coalescent droplet, water and heptane

Similar to the collisions of alkanes versus alkanes, fine spots appeared immediately after the collision between water and alkane droplets, and air bubbles were trapped within the contact surface.

\subsection{Immiscible Fuels: Alkanes vs. Methanol/Ethanol Collisions}

The droplet collisions between two immiscible fuels, i.e. methanol versus hexadecane, dodecane, decane, and heptane, and ethanol versus hexadecane, were tested in the study.

Similar to that in previous report [21], the results verified and further demonstrated that stable coalescent droplets could be obtained for immiscible alkanes and methanol, as well as for hexadecane and ethanol fuels. For head-on collision of the immiscible droplets, within the test ranges, say, 0.3 to $4.0 \mathrm{~m} / \mathrm{sec}$ of the collision velocity and 170 to $380 \mu \mathrm{m}$ of the droplet size, the stable coalescent droplets all appeared in the adhesive mode. In these situations, the two colliding droplets were in contact with each other, such that a contact surface could be clearly observed between the two immiscible components. The temporary coalescence followed by separation could occur as a result of sufficient increasing of the off-center distance at a given collision velocity.

Figures 8(a) and 8(b) show the photographic collision sequences of the permanent coalescence between decane and methanol droplets, with head-on and offcenter collisions, respectively. The results of methanol versus other alkanes and ethanol/hexadecane are quite similar, in which the appearance of the final coalescent droplets does not show much difference.

Fine spots, which are possibly trapped air bubbles, can also be observed for most of the tests.

\section{CONCLUDING REMARKS}

In this study, collisions between two different liquid droplets were conducted. The results of coalescent droplets lead to the following conclusions. 


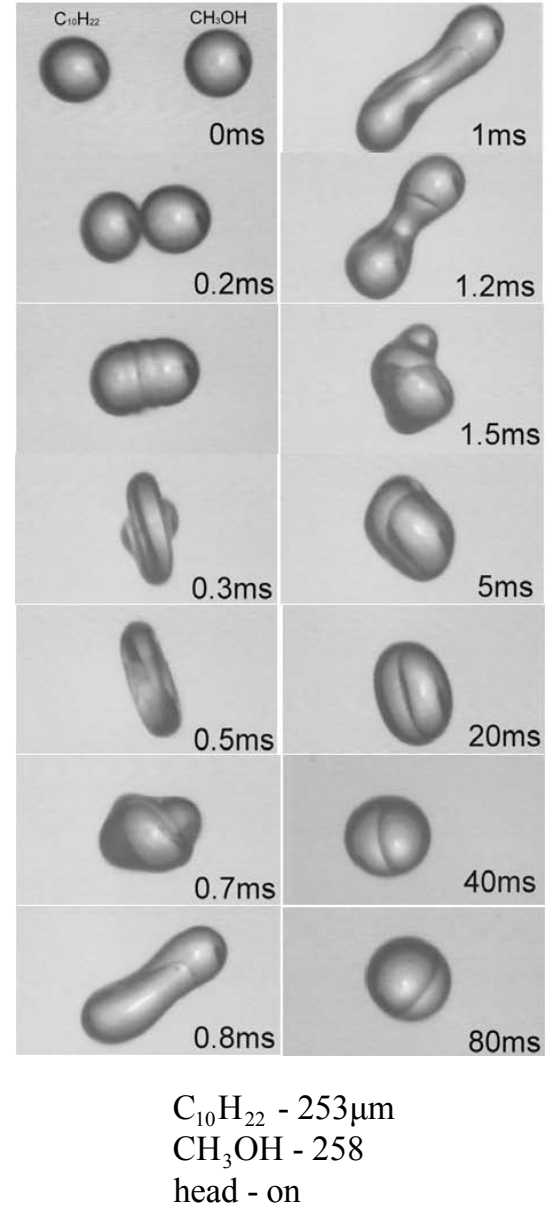

(a)

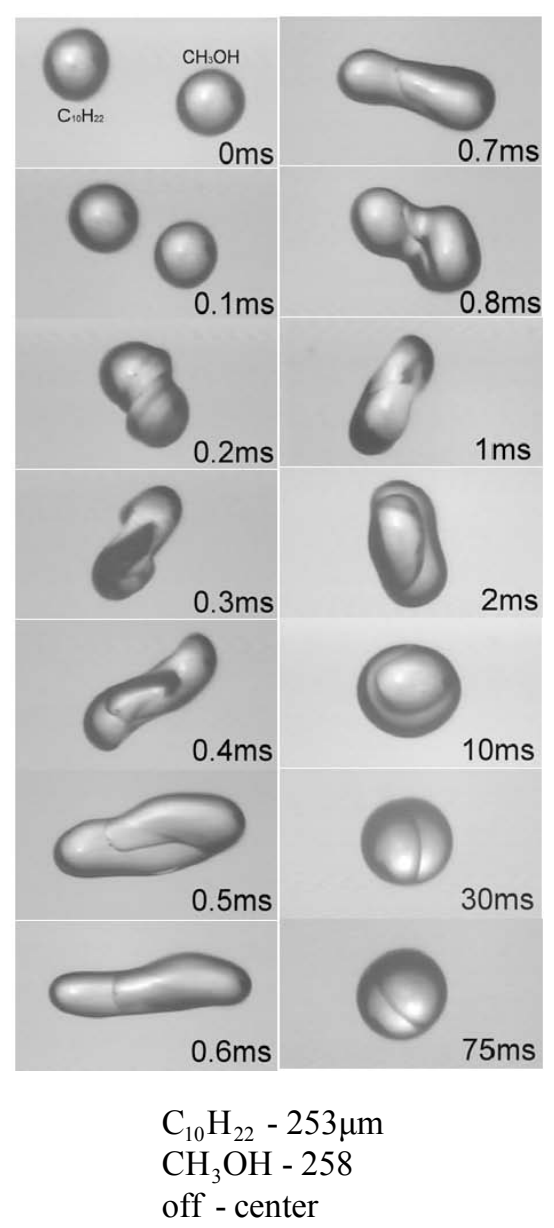

(b)

Fig. 8 Sequential photos of the formation of collision-coalescent droplet, decane and methanol

For miscible fuels, the two colliding droplets formed a spherical coalescent droplet within a short period of time. However, it took more time to have the components within the droplet to become uniform. In all cases, many fine spots appeared immediately after the collision, but only few or even none of the spots were left within the merged droplet after the completion of coalescent process. The entrapped spots were conjectured to be air bubbles, whose appearance depended on many factors, such as the relative collision velocity and/or orientation, and types of liquids of the two droplets.

In the collisions between immiscible water and alkane droplets, the droplets coalesced without mixing, and the coalescent droplet was either in insertive or adhesive mode. For most conditions, the insertive mode was prevalent. Adhesively coalescent droplets could only appear in collisions between water and hexadecane, at fairly low or sufficiently high collision velocities, or with increasing the off-center distance. However, by raising the environmental temperature, the adhesive coalescent droplet might change to the insertive coalescence mode. With sufficiently high water volume fraction of the coalescent droplet, the effect of surface tension can yield the breaking of the thin surface layer of alkane, and the enclosed water droplet thus are always partially exposed to the environment. The occurrence depends on the water volume fraction, the associated thickness of the thin surface layer, and the strength of surface tension of alkane. For the collisions between droplets of immiscible liquid fuels such as methanol and alkanes, and ethanol and hexadecane, only adhesive coalescent mode could exist under all the conditions. Air bubbles were also observed frequently after the completion of collision, which were trapped at the contact surface of the two immiscible fuels.

\section{ACKNOWLEDGEMENTS}

This work was supported by the National Science Council of Taiwan, Republic of China.

\section{REFERENCES}

1. Law, C. K., Prog. Energy Combustion Science, 8, p. 169 (1982).

2. Law, C. K. and Law, H. K., Modern Developments in Energy, Combustion, and Spectroscopy, F.A. 
Williams, A.K. Oppenheim, D.B. Olfe, and M. Lapp, Ed., Pergamon Press, p. 29 (1993).

3. Lasheras, J. C., Fernandez-Pello, A. C. and Dryer, F. L., Combust. Sci. Tech., 22, p. 195 (1980).

4. Sangiovanni, J. J. and Kesten, A. S., Combust. Sci. Tech., 16, p. 59 (1977).

5. Wang, C. H., Liu, X. Q. and Law, C. K., Combust. Flame, 56, p. 175 (1984).

6. Wang, C. H., Shy, K. H. and Lieu, L. C., Combust. Sci. Tech., 118, p. 63 (1996).

7. Wang, C. H. and Liou, D. S., J. Chinese Soc. of M.E. 17-4, p. 387 (1996).

8. Hopkinson, B. P., I. M. E. Proceedings, p. 679 (1913).

9. Greeves, G., Khan, I. M. and Onion, G., 16th International Symposium on Combustion, p. 321 (1976).

10. Cornet, I. and Nero, W. E., Industry and Engineering Chemistry, 2133 (1955).

11. Wang, C. H. and Ni, L. H., The Chinese J. Mechanics, 12-4, p. 465 (1996).

12. Ivanov, V. M. and Nefedov, P. I., NASA Tech. Translation TTF-258 (1965).

13. Lasheras, J. C., Fernandez-Pello, A. C. and Dryer, F. L., Combust. Sci. Tech., 21, p. 1 (1979).
14. Wang, C. H. and Law, C. K., Combust. Flame, 59, p. 53 (1985).

15. Wang, C. H. and Chen, J. T., Int. Comm. Heat Mass Transfer, 23, p. 823 (1996).

16. Ashgriz, N. and Givi, P., Int. J. Heat Fluid Flow, 8-3, p. 205 (1987).

17. Ashgriz, N. and Poo, J. Y., J. Fluid Mech., 221, p. 183 (1990).

18. Jiang, Y. J., Umemura, A. and Law, C. K., J. Fluid Mech., 234, p. 171 (1992).

19. Wang, C. H., Hung, W. G., Fu, S. Y., Huang, W. C. and Law, C. K., Combust. Flame, 114, p. 280 (2003).

20. Wang, C. H., Lin, C. Z., Hung, W. G., Huang, W. C. and Law, C. K., Combust. Sci. Tech., 176, p. 71 (2004).

21. Wang, C. H., Fu, S. Y., Kung, L. J. and Law, C. K., Proceedings of the Combustion Institute, 30, p. 1965 (2005).

(Manuscript received August 16, 2005, accepted for publication November 17, 2006.) 